\title{
Circuit motifs and graph properties of connectome development in C. elegans
}

\author{
Jordan K. Matelsky, Raphael Norman-Tenazas, Felicia Davenport, Elizabeth P. Reilly, William Gray-Roncal ${ }^{1}$
}

${ }^{1}$ Johns Hopkins Applied Physics Laboratory, Laurel, Maryland, United States

\begin{abstract}
Network science is a powerful tool that can be used to better explore the complex structure of brain networks. Leveraging graph and motif analysis tools, we interrogate $C$. elegans connectomes across multiple developmental time points and compare the resulting graph characteristics and substructures over time. We show the evolution of the networks and highlight stable invariants and patterns as well as those that grow or decay unexpectedly, providing a substrate for additional analysis.
\end{abstract}

connectomics | graph theory | development | motifs

Correspondence: \{jordan.matelsky,william.gray.roncal\}@jhuapl.edu

\section{Introduction}

As new synapse-resolution connectomics datasets are generated at increasing spatial volume extents, the neuroscience community is now faced with the exciting challenge of interpreting and exploring datasets that are much too large and numerous to manually proofread or inspect at the neuronlevel. Network analysis tools are one powerful strategy because they enable us to interpret the brain as a graph, where neurons are represented by nodes, and synapses are represented by directed edges. This simplified representation of connectivity provides one approach for summarizing important graph circuits and network properties. We leverage existing technologies and software, such as graph analysis toolkits and graph databases, in order to study the brain for advances in computation, health, and disease (1-3).

The $C$. elegans nematode serves as a well-studied, small nervous system thought to be highly conserved across isogenic individuals. Recent efforts (4) have mapped the complete $C$. elegans "wiring diagram," or connectome, of individuals at different ages, which provides us with a rich and useful set of graphs for comparison. This multi-individual dataset is a powerful tool to understand the development, modularity, and structure of a complete connectome throughout maturation and across a population, foreshadowing future studies in larger organisms such as Drosophila, mice, and humans. We leverage network science approaches in order to better characterize the types of modifications that the $C$. elegans brain undergoes throughout development.

\section{Results}

We identified and leveraged several graph analysis tools commonly used by the community to evaluate and explore connectome networks. We performed both computationallyinexpensive graph summary analyses, as well as more computationally-expensive subgraph structure analyses in order to better understand local connectome graph topology.
We share a selection of reproducible and noteworthy findings on seminal nematode connectomes from recent years $(4,5)$.
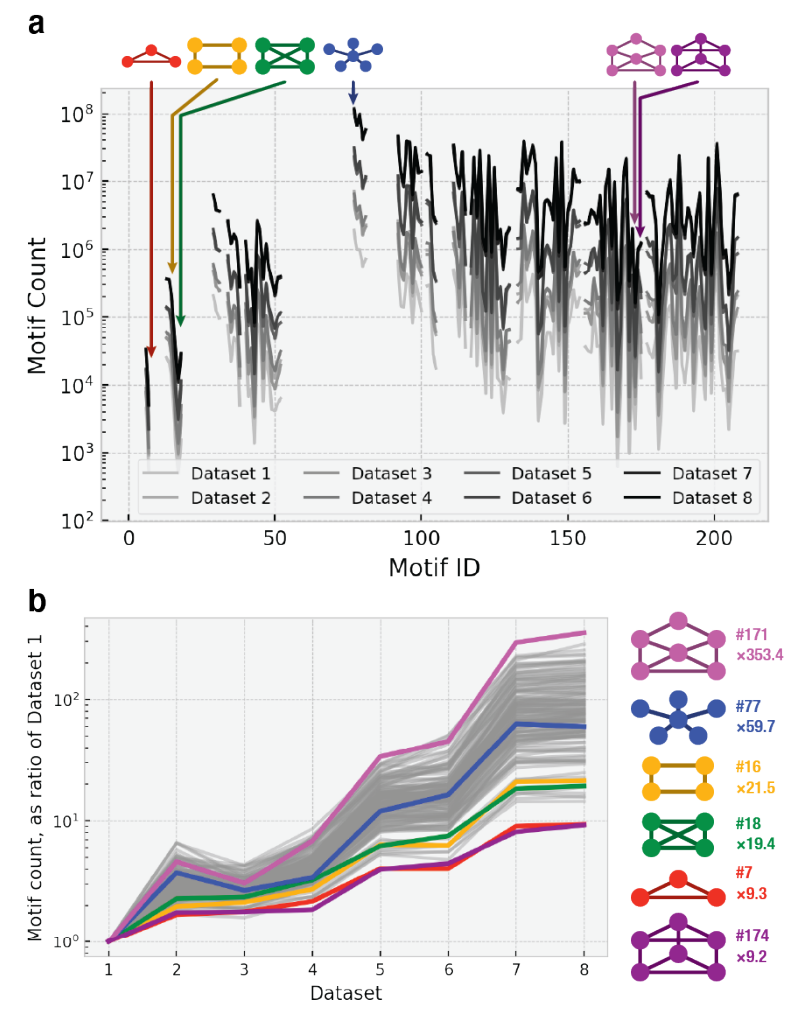

Fig. 1. Connectome motif growth over the lifespan of C. elegans. a. Undirected motif prevalence, showing all undirected graphs with six or fewer neurons. Six graphs of interest are identified along the $x$ axis. b. Motif growth throughout development. Motif counts are shown as a ratio of counts in Dataset 1: That is, a $y$-value of 2 indicates that there are twice as many of that motif as there were in Dataset 1. All undirected motifs from panel a are shown in grey. The six motifs of interest are rendered in color. The most rapidly growing motif (magenta) occurs 353 times more frequently in Dataset 8 as in Dataset 1. The most slowly growing motifs are triangles (red) and the triangular prism net (purple), at $9.24 \times$ and $9.33 \times$ prevalence in Dataset 8 . The motifs shown are identified in the Atlas of Graphs (6) as IDs $171,77,16,18,7$, and 174 (from top to bottom).

Undirected motif atlas scans of the growing $C$. elegans connectome reveal a changing local structure. We replicated the motif atlas-scan methods previously applied to partial mouse and invertebrate connectomes (7). This atlas-scan procedure involves searching for and counting all undirected subgraphs up to a certain size (here, subgraphs with six vertices or fewer). There are 210 such graphs. Fig. 1 shows the absolute motif counts of each motif for each of the eight datasets in (4).

We then related the motif prevalence at each timestep to the prevalence at the first timestamp, Dataset 1 (Fig. 1b). This 


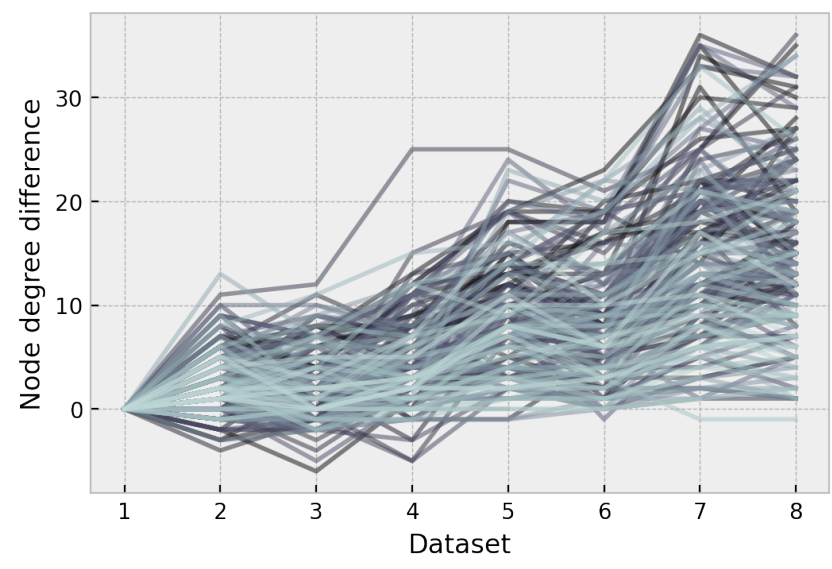

Fig. 2. Change in node degree. All changes in node degree are plotted as a function of animal age. The starting degree is defined as the node degree in Dataset 1 (4). The $y$-value is defined as the change in node degree since Dataset 1.

gives us a measure of the growth of motifs over developmental time. We call particular attention to the slowest- and fastest-growing motifs, as well as other motifs of interest. Using IDs from the Atlas of Graphs (6): Motifs \#174 and $\# 7$, the triangular prism net and the triangle graph, are the slowest-growing motifs, with only a nine-fold increase in motif prevalence. Motif \#171 is the fastest-growing motif, with more than a 350-fold increase in motif prevalence between datasets 1 and 8 . This is particularly notable in light of the substantial structural similarities between this motif and the slowest-growing \#174. Motif \#77, the motif with the highest absolute count at all eight timesteps, is of average growthrate, suggesting that absolute motif count and motif growth rate are not closely related measures.

Neuron degree increases during development. The average and maximum degree of a neuron in the $C$. elegans connectome tend to increase with animal age (Fig. 3). This is in contrast with the pruning process known to be common to vertebrate connectomes, in which high-degree neurons eliminate unnecessary synapses during early development. Because animal age and certain connectome statistics — such as maximum neuron degree or edge count - appear to be highly correlated, it may be possible to estimate such properties from an animal age alone. All neurons' degrees increase with age (Fig. 2). The only cell with a final degree smaller than its respective degree in Dataset 1 is GLRVL, a musclederived glial cell (9) - a result that may be attributable to biological noise.

Graph properties of a growing brain. (Table 1). We confirm the assertion in Ref. (4) that the adult graphs from Ref. (5) do not have the same properties as the recent adult graphs in Ref. (4). Furthermore, we also confirm that the two new adult datasets (4) have distinct graphs from each other, with distinct graph properties.

The $C$. elegans connectome is dominated by a single strongly connected component. The $C$. elegans connectome is composed of a dense network with inputs predominantly from sensory neurons and outputs predominantly to motor neurons. The (directed) density of the connectomes in Ref. (4) doubles from 0.245 (Dataset 1) to 0.489 and 0.502 in Datasets 7 and 8 . The largest strongly connected component contains nearly all neurons, regardless of animal age.

The adult connectome has significant variability in loop counts. Previous work $(10,11)$ has suggested that loops and cycles are important for computation in the growing nematode connectome. Here, we use our reproducible graph techniques to quantify this property during development, and show that the total number of loops tends to grow as the animal matures.

Degree distribution is nonrandom. We compared degree distributions between connectomes, as well as between connectomes and an Erdôs-Rényi random graph, following the strategy employed in a recent analysis (2). We show that none of the connectome variants have a degree distribution similar to that of a random graph (Fig. 4) by using a KolmogorovSmirnov test $(p \approx 0)$.

\section{Methods}

Taking advantage of previously-developed graph analysis frameworks (7), we computed motif searches (i.e., subgraph isomorphism counts), highlighting the prevalence of interesting structures over development. To understand the macro-structure of the C. elegans connectome networks during growth, we employed computationally inexpensive summary statistics, which are increasingly common in the network neuroscience community (12). Despite their apparent simplicity, these measures enable us to better understand the structure and nature of a brain during development.

We computed connectome graph analyses and summary statistics, such as those in Table 1, using a scalable network science toolkit developed to operate efficiently on thousandto billion-edge connectomes. For source code, see Supplemental Materials. All analyses were performed on consumer laptop hardware and can scale easily to compute and cluster resources for larger datasets.

\section{Discussion}

We can rapidly distill and analysis key network properties from complex datasets across an organisms development, using scalable, reproducible tools. Future work will include the analysis of sub-circuits within the connectome to see how their structure and function develop over time. An analysis using the volumetric image and segmentation data (4) could further elaborate on differences between adult connectomes and provide more insight into where and how the connectome differs between isogenic individuals.

It is interesting that different undirected subgraphs undergo different rates of growth in the maturing connectome. What biological mechanisms cause such dramatic differences in motif growth-rate? This points to a "preference" for certain structural properties in immature connectomes, and different local structural property preferences in a mature connectome - compatible with parallel observations on topological changes to a connectome during maturation (11). 
bioRxiv preprint doi: https://doi.org/10.1101/2021.07.11.451911; this version posted July 12, 2021. The copyright holder for this preprint (which was not certified by peer review) is the author/funder, who has granted bioRxiv a license to display the preprint in perpetuity. It is made available under aCC-BY-NC-ND 4.0 International license.


Fig. 3. a. The average node degree of a connectome increases with age, and is variable across adults. Orange lines denote original datasets for which a precise age was not provided (5). b. Node and edge counts in all individuals. c. Growth of new neurons over time.
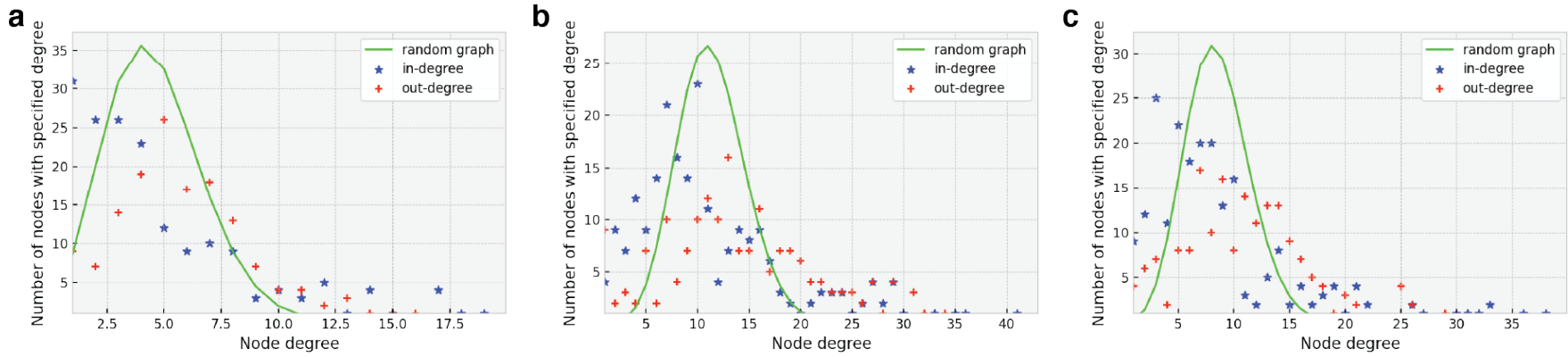

Fig. 4. In and out degree frequency distribution of selected connectomes. Random graph is created with the Erdős-Rényi model (8), where the probability of connection is equal to the compared graph's density. a. L1 larvae (4). b. Adult connectome (4). c. Adult connectome (5). In the adult connectomes, we see that there are a significant number of low-degree and high-degree nodes than random.

\begin{tabular}{|c|c|c|c|c|c|c|c|c|c|c|}
\hline Dataset & Nodes & Edges & Loops & Leaves & Max Degree & Mean Degree & Max SCC & Larval Stage & Age (Hours) & Source \\
\hline Witvliet 2020 Dataset 1 & 187 & 849 & 2 & 10 & 33 & 9.08 & 121 & L1 & 0 & (4) \\
\hline Witvliet 2020 Dataset 2 & 194 & 1095 & 2 & 8 & 36 & 11.28 & 135 & L1 & 5 & (4) \\
\hline Witvliet 2020 Dataset 3 & 198 & 1101 & 2 & 17 & 38 & 11.12 & 138 & L1 & 8 & (4) \\
\hline Witvliet 2020 Dataset 4 & 204 & 1324 & 3 & 11 & 43 & 12.98 & 149 & L1 & 16 & (4) \\
\hline Witvliet 2020 Dataset 5 & 211 & 1763 & 6 & 5 & 48 & 16.71 & 161 & $\mathrm{~L} 2$ & 25 & (4) \\
\hline Witvliet 2020 Dataset 6 & 216 & 1707 & 2 & 5 & 49 & 15.80 & 160 & L3 & 27 & (4) \\
\hline Witvliet 2020 Dataset 7 & 222 & 2450 & 16 & 1 & 58 & 22.07 & 191 & Adult & 50 & (4) \\
\hline Witvliet 2020 Dataset 8 & 219 & 2416 & 8 & 2 & 55 & 22.06 & 174 & Adult & 50 & (4) \\
\hline White 1986 JSH & 215 & 1725 & 2 & 2 & 48 & 16.04 & 168 & L4 & - & (5) \\
\hline White 1986 N2U & 221 & 1855 & 2 & 7 & 44 & 16.78 & 170 & Adult & - & (5) \\
\hline
\end{tabular}

Table 1. Graph Properties. A table of graph summary statistics for ten connectomes of interest. There are no orphan nodes and no lone pairs. All graphs were weakly connected. Max SCC refers to the number of nodes in the largest strongly connected component.

We hope this approach may serve as a model for the study of larger and less well-understood connectomes, such as emergent fruit-fly, rodent, and human connectomes (13-16).

\section{Supplemental Materials}

Motif-search tools are available at github.com/ aplbrain/dotmotif. Graph algorithm scaling tools are available at github.com/aplbrain/grand. Other software and data will be available upon publication.

\section{ACKNOWLEDGEMENTS}

Research reported in this publication was supported by the National Institute of Mental Health of the National Institutes of Health under Award Numbers R24MH114799 and R24MH114785. The content is solely the responsibility of the authors and does not necessarily represent the official views of the National Institutes of Health. This work was supported by JHU/APL Internal Research Funding.

\section{Bibliography}

1. Jody Clements, Tom Dolafi, Lowell Umayam, Nicole L Neubarth, et al. neuprint: Analysis tools for em connectomics. bioRxiv, 2020.

2. Louis K Scheffer. Graph properties of the adult Drosophila central brain. bioRxiv, 2020.

3. Elizabeth P. Reilly, Jeffrey S. Garretson, William R. Gray Roncal, Dean M. Kleissas, et al. Neural reconstruction integrity: A metric for assessing the connectivity accuracy of reconstructed neural networks. Frontiers in Neuroinformatics, Nov 2018.
4. Daniel Witvliet, Ben Mulcahy, James K. Mitchell, Yaron Meirovitch, et al. Connectomes across development reveal principles of brain maturation. bioRxiv, Apr 2020.

5. John G White, Eileen Southgate, J Nichol Thomson, Sydney Brenner, et al. The structure of the nervous system of the nematode Caenorhabditis elegans. Philos Trans $R$ Soc Lond B Biol Sci, 314(1165):1-340, 1986.

6. Ronald C Read and Robin J Wilson. An atlas of graphs. Oxford University Press, Inc., 2005

7. Jordan K. Matelsky, Elizabeth P. Reilly, Erik C. Johnson, Jennifer Stiso, et al. DotMotif: an open-source tool for connectome subgraph isomorphism search and graph queries. Scientific Reports, Jun 2021.

8. Paul Erdos, Alfréd Rényi, et al. On the evolution of random graphs. Publ. Math. Inst. Hung Acad. Sci, 5(1):17-60, 1960.

9. Grigorios Oikonomou and Shai Shaham. The glia of caenorhabditis elegans. Glia, 59(9): 1253-1263, Nov 2010. ISSN 0894-1491. doi: 10.1002/glia.21084.

10. Ann E Sizemore, Chad Giusti, Ari Kahn, Jean M Vettel, et al. Cliques and cavities in the human connectome. Journal of computational neuroscience, 2018.

11. Alec Helm, Ann S. Blevins, and Danielle S. Bassett. The growing topology of the c. elegans connectome, 2020

12. Louis K Scheffer, C Shan Xu, Michal Januszewski, Zhiyuan Lu, et al. A connectome and analysis of the adult Drosophila central brain. Elife, 9:e57443, 2020

13. Casey M. Schneider-Mizell, Agnes L. Bodor, Forrest Collman, Derrick Brittain, et al. Chandelier cell anatomy and function reveal a variably distributed but common signal. biorXiv, April 2020. doi: 10.1101/2020.03.31.018952.

14. Sven Dorkenwald, Nicholas L. Turner, Thomas Macrina, Kisuk Lee, et al. Binary and analog variation of synapses between cortical pyramidal neurons. biorXiv, December 2019.

15. C. Shan Xu, Michal Januszewski, Zhiyuan Lu, Shin-ya Takemura, et al. A Connectome of the Adult Drosophila Central Brain. biorXiv, Jan 2020. doi: 10.1101/2020.01.21.911859.

16. Alexander Shapson-Coe, Michał Januszewski, Daniel R. Berger, Art Pope, et al. A connectomic study of a petascale fragment of human cerebral cortex. bioRxiv, 2021. 